\title{
Integer Cech Cohomology of Icosahedral Projection Tilings
}

\author{
F. Gähler* \\ Institut für Theoretische und Angewandte Physik, \\ Universität Stuttgart, D-70550 Stuttgart, Germany \\ J. Hunton \\ Department of Mathematics, University of Leicester, Leicester, LE1 7RH, United Kingdom \\ J. Kellendonk \\ Université de Lyon, Université Lyon 1, CNRS, UMR5208, Institut Camille Jordan, \\ 43 blvd du 11 novembre 1918, F-69622 Villeurbanne cedex, France
}

\begin{abstract}
The integer Cech cohomology of canonical projection tilings of dimension three and codimension three is derived. These formulae are then evaluated for several icosahedral tilings known from the literature. Rather surprisingly, the cohomologies of all these tilings turn out to have torsion. This is the case even for the Danzer tiling, which is, in some sense, the simplest of all icosahedral tilings. This result is in contrast to the case of two-dimensional canonical projection tilings, where many examples without torsion are known.
\end{abstract}

\section{INTRODUCTION}

The local isomorphism class (LI class) of a repetitive tiling can be given a metric, which makes it a compact topological space of tilings, called the hull of the tiling. Many important properties of the tiling depend on the topology of its hull, which can be characterized to some extent by topological invariants. For a brief introduction to the topology of tiling spaces, and why it matters, we refer to [1]. The simplest topological invariants are the Cech cohomology groups, which moreover are practically computable for large classes of tilings. This relies mainly on the fact that tiling spaces are inverse limits of simpler spaces 22. Using such an inverse limit construction, Anderson and Putnam [3] gave a practical algorithm to compute the Cech cohomology of primitive substitution tilings, an algorithm which works for integer, rational, or any other kind of coefficients. Another important class of tilings whose cohomology is computable are canonical projection tilings, with a definition of 'canonical' [4] which is actually more general than the usual one. Basically, one only has to assume polyhedral windows whose faces satisfy certain rationality conditions. For such canonical projection tilings, the cohomology with rational coefficients is determined by the formalisms of Forrest, Hunton and Kellendonk (FHK) [4, 5] and of Kalugin [6]. For the co-dimension 2 case, even the integer result was implicitly contained in [4], but the torsion part remained unnoticed because it was thought [4, 5] that the cohomology of canonical projection tilings was always free. This turned out to be incorrect, however, and was disproved by counterexamples [7]. Whereas the co-dimension 2 case is therefore easy to extend to integer coefficients (also in Kalugin's formalism), the co-

\footnotetext{
* Present address: Fakultät für Mathematik, Universität Bielefeld, Postfach 1001 31, D-33615 Bielefeld, Germany

Email: gaehler@math.uni-bielefeld.de
}

dimension 3 case had resisted any such attempt for a long time. Recent progress enables us, however, to overcome these difficulties in both formalisms. The refined theories of FHK and Kalugin both allow now to determine the cohomology with integer coefficents for a large number of examples, even though there remain cases where the torsion part cannot be fully determined. In this paper, we sketch the essential steps and ingredients of the computation for an integer version of Kalugin's formalism, whose setup is somewhat easier to explain. The results for a number of well-known icosahedral tilings from the literature are then presented. It turns out that all these tilings have torsion in their integer Cech cohomology. For a more detailed description, and for an integer version of the FHK formalism, we refer to a forthcoming publication [8].

\section{CANONICAL PROJECTION TILINGS}

The tilings we consider here are known as canonical projection tilings, where we use the broader, more general notion of 'canonical' from [4], rather than the traditional one used in [5]. Despite their name, canonical projections tilings are more conveniently described as sections through a higher-dimensional periodic tiling. For this purpose, we consider a Euclidean space $E^{n}=E^{\|} \oplus E^{\perp}$, containing a lattice $\Gamma$ of full rank $n$, whose projection on either summand is both bijective and dense. In $E^{n}$, we have a $\Gamma$-periodic tiling, whose tiles $K_{i}$ are prisms $K_{i}=K_{i}^{\|} \times K_{i}^{\perp}$. Generic sections parallel to $E^{\|}$yield then tilings of $E^{\|}$by tiles $K_{i}^{\|}$. Each tile $K_{i}^{\|}$has an acceptance domain $K_{i}^{\perp} \subset E^{\perp}$ : whenever the section touches it, the tile is present in the tiling. Sections touching the boundary of an acceptance domain are called singular; they correspond to more than one tiling. The periodic tiling can be wrapped on the $n$-torus $T^{n}=E^{n} / \Gamma$. Disregarding singular sections, the points in this torus label the possible tilings in our tiling space $\Omega$. This is known as the torus parametrization [9]. 
In the following, we consider tilings whose acceptance domains are polyhedral. If a section touches a face $F$ in the boundary of an acceptance domain $K^{\perp}$, the corresponding tiling is not unique in the set $F \times K^{\|}$(which has codimension 1). Often, more than one tile is singular at a time. The ambiguity of a singular section has to be resolved by cutting up the torus along the singular set where the tiling is ambiguous, and completing it from both sides. However, a point in the cut-up torus still does not necessarily correspond to a non-singular cut. The tiling may still be singular somewhere away from the origin. To avoid this, one would have to cut up the torus along the whole hyperplane $F \times E^{\|}$. Kalugin [6] has proposed to proceed in steps, using an inverse limit construction: at each step, the torus is cut up only by a finite amount. One thereby arrives at a sequence of tori $\Omega_{r}=T^{n} \backslash A_{r}$, cut-up along larger and larger sets $A_{r}$. Kalugin has shown that the full tiling space $\Omega$ is given by the inverse limit of this sequence of cut-up tori $\Omega_{r}$. Moreover, their cohomology satisfies the exact sequence

$$
\begin{aligned}
\rightarrow H^{k}\left(\Omega_{r}\right) \rightarrow & H_{n-k-1}\left(A_{r}\right) \\
& \rightarrow H_{n-k-1}\left(T^{n}\right) \rightarrow H^{k+1}\left(\Omega_{r}\right) \rightarrow
\end{aligned}
$$

Next, we make use of the fact that canonical projection tilings have acceptance domains with rationally oriented faces (with respect to the projected lattice $\Gamma^{\perp}$ ). For the icosahedral tilings considered here, for which $n=6$, this implies that any component $F \times K^{\|}$of the singular set $A_{r}$ is contained in a 5-dimensional hyperplane comprising 4 independent lattice directions. This set is therefore 4-periodic, containing whole 4-dimensional arrays of sets $F \times K^{\|}$, and if $r$ is increased, these pieces grow together to form, for some finite $r$, a full, rational 4-plane thickened along a fifth direction. Actually, this thickened rational 4-plane is wrapped onto the torus, and the set $A_{r}$ is therefore a finite union of rational 4-tori, each thickened along a fifth direction. The homotopy type of $A_{r}$ (and thus its homology) does not change if $r$ is further increased. Moreover, it is often possible to find a finite arrangement $\tilde{A}$ of normal, thin 4-tori, which is homotopy equivalent to $A_{r}$, and thus has the same homology (see [6]). For all the 3-dimensional icosahedral tilings considered below, this is the case, and we can work with such a set $\tilde{A}$, which consists of a union of $L_{2} 4$-tori $T^{\alpha}$, indexed by $\alpha \in I_{2}$, each of which is stabilized by the translations in a sublattice $\Gamma^{\alpha}$ of rank 4 . These 4-tori intersect in $L_{1} 2$-tori $T^{\theta}$, indexed by $\theta \in I_{1}$, each stabilized by a sublattice $\Gamma^{\theta}$ of rank 2 , and $L_{0}$ intersection points. The $L_{1}^{\alpha} 2$-tori contained in $T^{\alpha}$ are indexed by $I_{1}^{\alpha}$, the $L_{0}^{\alpha}$ intersection points in $T^{\alpha}$ by $I_{0}^{\alpha}$, and the $L_{0}^{\theta}$ intersection points in $T^{\theta}$ by $I_{0}^{\theta}$. Even though the set $\tilde{A}$ to be removed from the torus $T^{6}$ is already reasonably simple, we have to make one more replacement by yet another homotopy equivalent set: we pass from $\tilde{A}$ to its simplicial resolution $A$ (see [6]). The set $A$ is the union of a still larger number of spaces, which comprises also spaces homotopic to the former intersection tori. The advantage of this is, that the maximal number of spaces with a common intersection is drastically reduced, which proves useful in the computation of the homology of $A$ via a Mayer-Vietoris spectral sequence.

\section{COHOMOLOGY COMPUTATION}

We have now seen that the singular set $A_{r}$ is, for sufficiently large $r$, homotopy equivalent to the set $A$, which can therefore replace $A_{r}$ in the exact sequence (10), and after the disappearance of $r$ the direct limit of the cohomology of $\Omega_{r}$ is easily carried out. Moreover, the sequence (11) can be split as follows:

$$
\begin{aligned}
0 \rightarrow S_{k}(R) & \rightarrow H^{k}(\Omega ; R) \rightarrow H_{6-k-1}(A ; R) \\
& \stackrel{\alpha_{R}^{k+1}}{\longrightarrow} H_{6-k-1}\left(T^{6} ; R\right) \rightarrow S_{k+1}(R) \rightarrow 0
\end{aligned}
$$

where we have explicitly indicated the coefficient group $R$. To determine $H^{k}(\Omega ; R)$, we need to compute $H_{*}\left(T^{6} ; R\right), H_{*}(A ; R)$, and $S_{k}(R)=\operatorname{coker} \alpha_{R}^{k}$ for the different coefficient groups $R=\mathbb{Q}, \mathbb{Z}$, or $\mathbb{F}_{p}$. A few conclusions on $S_{k}(R)$ can immediately be drawn. Since $H^{k}(\Omega ; R)=0$ for $k>3$, we have $S_{k}(R)=0$ for $k>3$, and $\alpha_{R}^{4}$ is onto. Also, because $A$ has dimension 4 , we get $S_{k}(R)=H_{6-k}\left(T^{6} ; R\right)$ for $k<2$, and since the constituent spaces of $A$ have intersections of dimension at most $2, S_{2}(R)$ is also easily computed: $S_{2}(R)=\Lambda_{4} \Gamma \otimes R /\left\langle\Lambda_{4} \Gamma^{\alpha} \otimes R\right\rangle$, where $\Lambda_{k} \Gamma$ denotes the $k$-fold totally antisymmetric tensor product of $\Gamma$.

Before we address the computation of $S_{3}(R)$, we determine the homology of $A$. As $A$ is a finite union of spaces $A_{i}$, its homology can be computed using a MayerVietoris spectral sequence (MVSS), which expresses the homology of $A$ in terms of the homology of the $A_{i}$ and their intersections. We use a homological version of the MVSS used in [10], where the reader can also find more information on the evaluation of the MVSS in a similar setting. It is important here that we have passed to the simplicial resolution $A$ of $\tilde{A}$, which drastically reduces the number of columns required in the MVSS (which is equal to the maximal number of spaces $A_{i}$ having a common intersection, compare [6]). The first page (see [10]) $E_{k, \ell}^{1}$ of the Mayer-Vietoris double complex contains the direct sums of the $\ell$-homologies of all intersections of $k$ constituent spaces $A_{i}$ of $A$. It is given in Table \ All these groups are free, and we also know that $H_{0}(A)=R$ and $H_{1}(A)=R^{6}$. As a consequence, the total rank of all differentials in this and the second page, departing from the diagonal $k+\ell=2$, can be computed. Beyond this total rank, explicit knowledge of those differentials is not needed. Only one differential needs to be known explicitly:

$$
d_{1,2}^{1}: \bigoplus_{\alpha \in I_{2}} \oplus_{\theta \in I_{1}^{\alpha}} \Lambda_{2} \Gamma^{\theta} \rightarrow\left(\oplus_{\alpha \in I_{2}} \Lambda_{2} \Gamma^{\alpha}\right) \oplus\left(\oplus_{\theta \in I_{1}} \Lambda_{2} \Gamma^{\theta}\right)
$$

Its source space is embedded in the obvious way in both sectors of the target space, with different signs. The cokernel of the differential $d_{1,2}^{1}$ is the only possible source 


\begin{tabular}{|c|c|c|}
\hline$\oplus_{\alpha \in I_{2}} \Lambda_{4} \Gamma^{\alpha}$ & & \\
\hline$\oplus_{\alpha \in I_{2}} \Lambda_{3} \Gamma^{\alpha}$ & & \\
\hline$\oplus_{\alpha \in I_{2}} \Lambda_{2} \Gamma^{\alpha} \oplus \oplus_{\theta \in I_{1}} \Lambda_{2} \Gamma^{\theta}$ & $\oplus_{\alpha \in I_{2}} \oplus_{\theta \in I_{1}^{\alpha}} \Lambda_{2} \Gamma^{\theta}$ & \\
\hline$\oplus_{\alpha \in I_{2}} \Lambda_{1} \Gamma^{\alpha} \oplus \oplus_{\theta \in I_{1}} \Lambda_{1} \Gamma^{\theta}$ & $\oplus_{\alpha \in I_{2}} \oplus_{\theta \in I_{1}^{\alpha}} \Lambda_{1} \Gamma^{\theta}$ & \\
\hline $\mathbb{Z}^{L_{2}} \oplus \mathbb{Z}^{L_{1}} \oplus \mathbb{Z}^{L_{0}}$ & $\oplus_{\alpha \in I_{2}} \mathbb{Z}^{L_{1}^{\alpha}} \oplus_{\alpha \in I_{2}} \mathbb{Z}^{L_{0}^{\alpha}} \oplus \oplus_{\theta \in I_{1}} \mathbb{Z}^{L_{0}^{\theta}}$ & $\oplus_{\alpha \in I_{2}} \oplus_{\theta \in I_{1}^{\alpha}} \mathbb{Z}^{L_{0}^{\theta}}$ \\
\hline
\end{tabular}

TABLE I: First page of the Mayer-Vietoris spectral sequence for the homology of $A$.

of torsion in $H_{*}(A ; \mathbb{Z})$. As it appears in the leftmost column of the double complex, the homology of $A$ is still the direct sum of $E_{k, \ell}^{\infty}, n=k+\ell$, despite the presence of torsion. The final result on the homology of $A$ is summarized in Theorem 1 .

Theorem 1 Suppose $A$ is an arrangement of singular tori as above. For any coefficient group $R=\mathbb{Q}, \mathbb{Z}$, or $\mathbb{F}_{p}$, we then have:

$$
\begin{aligned}
H_{0}(A ; R) & =R \\
H_{1}(A ; R) & =R^{6} \\
H_{2}(A ; R) & =\text { coker } d_{1,2}^{1}(R) \oplus R^{f} \\
H_{3}(A ; R) & =\left(\oplus_{\alpha \in I_{2}} \Lambda_{3} \Gamma^{\alpha}\right) \oplus\left(\operatorname{ker} d_{1,2}^{1}(R)\right) \\
H_{4}(A ; R) & =\oplus_{\alpha \in I_{2}} \Lambda_{4} \Gamma^{\alpha} \\
\text { with } \quad f & =-3 L_{2}-L_{1}+\sum_{\alpha \in I_{2}} L_{1}^{\alpha}+5+\chi \\
\text { and } \quad \chi & =L_{0}-\sum_{\alpha \in I_{2}} L_{0}^{\alpha}+\sum_{\alpha \in I_{2}} \sum_{\theta \in I_{1}^{\alpha}} L_{0}^{\theta}-\sum_{\theta \in I_{1}} L_{0}^{\theta}
\end{aligned}
$$

For $R=\mathbb{Z}$, the only potential source of torsion in $H_{*}(A ; \mathbb{Z})$ is coker $d_{1,2}^{1}(\mathbb{Z}) . \chi$ is the Euler characteristic of $\Omega$.

Next, we need to determine $S_{3}(R)$ for the different coefficient groups $R$ (all other $S_{k}(R)$ are already known). We start with $R=\mathbb{Q}$. For $S_{3}(\mathbb{Q})$ we need the image of $\alpha_{\mathbb{Q}}^{3}$, which is at least $M=\left\langle\Lambda_{3} \Gamma^{\alpha} \otimes \mathbb{Q}\right\rangle$. We claim that it cannot be bigger than $M$. To see this, consider a simplicial decomposition of $T^{6}$, containing $A$ as a subcomplex, and take the chain maps

$$
C_{3}(A) \stackrel{i}{\longrightarrow} C_{3}\left(T^{6}\right) \stackrel{P}{\longrightarrow} \Lambda_{3} \Gamma \otimes \mathbb{Q}
$$

$P$ assigns to each simplex its volume form. As $P$ vanishes on boundaries, it induces an isomorphism $H_{3}\left(T^{6}\right) \rightarrow$ $\Lambda_{3} \Gamma \otimes \mathbb{Q}$. All cells in $A$ map under $P$ to directions contained in $M$, and so the image of $\alpha_{\mathbb{Q}}^{3}$ cannot be larger than $M$. The rational result gives us bounds on the possible values of $\operatorname{Im} \alpha_{\mathbb{Z}}^{3}$ :

$$
M_{1}=\left\langle\Lambda_{3} \Gamma^{\alpha}\right\rangle \subseteq \operatorname{Im} \alpha_{\mathbb{Z}}^{3} \subseteq\left\langle\Lambda_{3} \Gamma^{\alpha} \otimes \mathbb{Q}\right\rangle \cap \Lambda_{3} \Gamma=M_{2}
$$

If $M_{1}=M_{2}, S_{3}(\mathbb{Z})=$ coker $\alpha_{\mathbb{Z}}^{3}=\Lambda_{3} \Gamma /\left\langle\Lambda_{3} \Gamma^{\alpha}\right\rangle$ is free, and we are done. Otherwise, we can try the sharper lower bound

$$
M_{1}^{\prime}=\left\langle b_{1}^{\theta} \wedge b_{2}^{\theta} \wedge v \mid \theta \in I_{1},\left\langle b_{1}^{\theta}, b_{2}^{\theta}\right\rangle=\Gamma^{\theta}, v \in \Gamma\right\rangle
$$

on $\operatorname{Im} \alpha_{\mathbb{Z}}^{3}$, which comes about as follows. The 3-chains in $C_{3}(A)$ need not be closed within a single constituent torus of $A$. Rather, the boundaries of 3-chains contained in different 4-tori may cancel each other. To see this, we unfold the torus for a moment, and consider two lattice 4 -planes $p_{1}$ and $p_{2}$, intersecting in a 2 -plane $\ell_{1}$ with stabilizer $\Gamma^{\theta}$. Let $v$ be any lattice vector. The planes $p_{1}$ and $p_{2}+v$ intersect in a plane $\ell_{2}$ parallel to $\ell_{1}$, and the planes $p_{1}+v$ and $p_{2}+v$ intersect in a plane $\ell_{3}=\ell_{1}+v$. We can now construct two 3 -chains, one contained in $p_{1}$ with boundary $\left[\ell_{2}\right]-\left[\ell_{1}\right]$, and one contained in $p_{2}$ with boudnary $\left[\ell_{3}\right]-\left[\ell_{1}\right]$. As $\ell_{1}$ and $\ell_{3}$ are equivalent in the torus, the boundaries of the two 3 -chains cancel, and we have a 3-cyle homologous to $b_{1}^{\theta} \wedge b_{2}^{\theta} \wedge v$, where $\left\{b_{i}^{\theta}\right\}$ is a basis of $\Gamma^{\theta}$; this proves the bound (3). Again, if $M_{1}^{\prime}=M_{2}$, $S_{3}(\mathbb{Z})=\operatorname{coker} \alpha_{\mathbb{Z}}^{3}=\Lambda_{3} \Gamma / M_{2}$ is free.

We now restrict ourselves to the case where $S_{3}(\mathbb{Z})$ is free. There may be still torsion in $H_{2}(A ; \mathbb{Z})$, however, and it is not clear yet whether it lifts to $H^{3}(\Omega ; \mathbb{Z})$. To see this, note that the sequence $0 \rightarrow \mathbb{Z} \rightarrow X \rightarrow \mathbb{Z}_{2} \rightarrow 0$ has two solutions, $X=\mathbb{Z}$ and $X=\mathbb{Z} \oplus \mathbb{Z}_{2}$. No integral arguments will help to distinguish these cases, and we need further input. It turns out that the knowledge of $S_{3}\left(\mathbb{F}_{p}\right)$ for suitable primes $p$ will help. Our aim is to show that if $S_{3}(\mathbb{Z})$ is free, then $r k\left(S_{3}(\mathbb{Q})\right)=r k\left(S_{3}\left(\mathbb{F}_{p}\right)\right)$. To do so, we write $A \rightarrow T^{6}$ as a composite:

$$
A \stackrel{\Delta}{\rightarrow} A \times \ldots \times A \stackrel{a_{1} \times \ldots \times a_{6}}{\longrightarrow} S^{1} \times \ldots \times S^{1} \stackrel{i}{\rightarrow} T^{6}
$$

In homology, we then get

$$
\begin{aligned}
H_{3}(A) \stackrel{\Delta *}{\longrightarrow} \oplus_{r_{1}+\cdots+r_{6}=3} H_{r_{1}}(A) \otimes \cdots \otimes & H_{r_{6}}(A) \\
\stackrel{a_{1} * \otimes \cdots \otimes a_{6} *}{\longrightarrow} \oplus_{r_{1}+\cdots+r_{6}=3} H_{r_{1}}\left(S^{1}\right) \otimes \cdots & \cdots H_{r_{6}}\left(S^{1}\right) \\
& \stackrel{i *}{\longrightarrow} H_{3}\left(T^{6}\right)
\end{aligned}
$$

Since $H_{k}\left(S^{1}\right)=0$ for $k \geq 2$, only $H_{0}(A)$ and $H_{1}(A)$ can contribute. For those dimensions, however, $H_{k}(A)$ is free; passing from $\mathbb{Q}$ to $\mathbb{F}_{p}$ can therefore not change the rank of $\operatorname{Im} \alpha^{3}$.

We are now going to compare the ranks of the groups in (2) for the different coefficient groups $\mathbb{Q}$ and $\mathbb{F}_{p}$. From the universal coefficient theorem we know that

$$
\begin{aligned}
& r k\left(H^{k}\left(\Omega ; \mathbb{F}_{p}\right)\right)-r k\left(H^{k}(\Omega ; \mathbb{Q})\right) \\
& \quad=T_{p}\left(H^{k}(\Omega ; \mathbb{Z})\right)+T_{p}\left(H^{k+1}(\Omega ; \mathbb{Z})\right), \\
& r k\left(H_{k}\left(A ; \mathbb{F}_{p}\right)\right)-r k\left(H_{k}(A ; \mathbb{Q})\right) \\
& \quad=T_{p}\left(H_{k}(A ; \mathbb{Z})\right)+T_{p}\left(H_{k-1}(A ; \mathbb{Z})\right),
\end{aligned}
$$




\begin{tabular}{|c|c|c|c|c|c|c|l|}
\hline$H^{3}$ & $H^{2}$ & $H^{1}$ & $H^{0}$ & $\chi$ & planes & $\Gamma$ & \\
\hline $\mathbb{Z}^{20} \oplus \mathbb{Z}_{2}$ & $\mathbb{Z}^{16}$ & $\mathbb{Z}^{7}$ & $\mathbb{Z}$ & 10 & 5-fold & $\mathrm{F}$ & Danzer \\
\hline $\mathbb{Z}^{181} \oplus \mathbb{Z}_{2}$ & $\mathbb{Z}^{72} \oplus \mathbb{Z}_{2}$ & $\mathbb{Z}^{12}$ & $\mathbb{Z}$ & 120 & mirror & $\mathrm{P}$ & Ammann-Kramer \\
\hline $\mathbb{Z}^{331} \oplus \mathbb{Z}_{2}^{20} \oplus \mathbb{Z}_{4}$ & $\mathbb{Z}^{102} \oplus \mathbb{Z}_{2}^{4} \oplus \mathbb{Z}_{4}$ & $\mathbb{Z}^{12}$ & $\mathbb{Z}$ & 240 & mirror & $\mathrm{F}$ & dual canonical $D_{6}$ \\
\hline $\mathbb{Z}^{205} \oplus \mathbb{Z}_{2}^{2}$ & $\mathbb{Z}^{72}$ & $\mathbb{Z}^{7}$ & $\mathbb{Z}$ & 145 & 3,5 -fold & $\mathrm{F}$ & canonical $D_{6}$ \\
\hline
\end{tabular}

TABLE II: Cohomology of icosahedral tilings from the literature. Also indicated are the Euler characteristic $\chi$, the type of icosahedral lattice ( $\mathrm{P}$ for primitive, $\mathrm{F}$ for face-centered), and the orientation of the boundaries of the acceptance domains (perpendicular to 3 -fold or 5-fold axes of the icosahedron, or parallel to its mirror planes).

where $T_{p}(X)$ is the rank of $p$-torsion in $X$. Clearly, we are interested mainly in those primes $p$ for which $H_{2}(A ; \mathbb{Z})$ has $p$-torsion. Inserting this into (2), and using $r k\left(S_{3}(\mathbb{Q})\right)=r k\left(S_{3}\left(\mathbb{F}_{p}\right)\right)$, we obtain

$$
\begin{gathered}
r k\left(H^{3}\left(\Omega ; \mathbb{F}_{p}\right)\right)-r k\left(H^{3}(\Omega ; \mathbb{Q})\right)=T_{p}\left(H_{2}(A ; \mathbb{Z})\right) \\
\operatorname{Torsion}\left(H^{3}(\Omega ; \mathbb{Z})\right)=\operatorname{Torsion}\left(H_{2}(A ; \mathbb{Z})\right)
\end{gathered}
$$

We therefore have arrived at our main result, which is summarized in Theorem 2 .

Theorem 2 Consider a dimension 3, co-dimension 3 canonical projection tiling with arrangement of singular tori $A$ as above, and suppose $S_{3}(\mathbb{Z})$ is free. For any coefficient field $R=\mathbb{Q}$ or $\mathbb{F}_{p}$, we then have

$$
\begin{aligned}
r k\left(H^{k}(\Omega ; R)\right)=r k\left(H_{6-k-1}\right. & (A ; \mathbb{Z}))+r k\left(S_{k}(R)\right) \\
& +r k\left(S_{k+1}(R)\right)-\left(\begin{array}{c}
6 \\
k+1
\end{array}\right) .
\end{aligned}
$$

For the coefficient ring $R=\mathbb{Z}$, the same relation holds for the free ranks, and

$$
\begin{aligned}
& \operatorname{Torsion}\left(H^{2}(\Omega ; \mathbb{Z})\right)=\operatorname{Torsion}\left(S_{2}(\mathbb{Z})\right) \\
& \operatorname{Torsion}\left(H^{3}(\Omega ; \mathbb{Z})\right)=\operatorname{Torsion}\left(H_{2}(A ; \mathbb{Z})\right)
\end{aligned}
$$

All other $\left.H^{k}(\Omega ; \mathbb{Z})\right)$ are free.

\section{RESULTS AND DISCUSSION}

We have evaluated the formulae from Theorems 1 and 2 for several icosahedral tilings from the literature, with the help of a computer. These tilings comprise the Danzer tiling [11], the Ammann-Kramer tiling [12], the canonical $D_{6}$ and the dual canonical $D_{6}$ 13]. The results are given in Table II. Most remarkably, all these tilings have torsion in $H^{3}(\Omega)$, whose origin is in the torsion of $H_{2}(A)$. Two examples have torsion also in $H^{2}(\Omega)$, resulting from the torsion in $S_{2}(\mathbb{Z})$. In two dimensions, at least the simpler canonical projection tilings have no torsion, so that it is rather surprising that even the Danzer tiling with its combinatorially simple set $A$ has torsion. It should be noted, however, that the torsion of the Danzer tiling has a different origin. It comes from the homology of $A$, which does not provide any torsion in the two-dimensional case.
It should also be remarked that $S_{3}$ is free for all these examples. Only for the dual canonical $D_{6}$ we had to resort to the sharper bound (3) to show this.

The methods presented here are applicable also in the 2-dimensional case, but everything is considerably simpler. Details on the 2-dimensional case will be presented elsewhere [8].

[1] Sadun, L.: Tilings, tilings spaces and topology. Phil. Mag. 86 (2006) 875-881.

[2] Sadun, L.: Tiling Spaces are Inverse Limits. J. Math. Phys. 44 (2003) 5410-5414.

[3] Anderson, J.E.; Putnam, I.F.: Topological invariants for substitution tilings and their associated $C^{*}$-algebras. Ergod. Th. \& Dynam. Syst. 18 (1998) 509-537.

[4] Forrest, A.H.; Hunton, J.R.; Kellendonk, J.: Cohomology of canonical projection tilings. Commun. Math. Phys. 226 (2002) 289-322.

[5] Forrest, A.H.; Hunton, J.R.; Kellendonk, J.: Topological invariants for projection method patterns. Mem. Amer. Math. Soc. 758 (2002).

[6] Kalugin, P.: Cohomology of quasiperiodic patterns and matching rules. J. Phys. A: Math. Gen. 38 (2005) 31153132.

[7] Gähler, F.: Lecture given at BIRS Workshop on Aperiodic Order: Dynamical Systems, Combinatorics, and Operators, Banff, June 2004.

[8] Gähler, F.; Hunton, J.R.; Kellendonk, J.: in preparation.

[9] Baake, M.; Hermisson, J.; Pleasants, P.A.B.: The torus parametrization of quasiperiodic LI-classes. J. Phys. A: Math. Gen. 30 (1997) 3029-3056.

[10] Basu, S.: Computing the Betti numbers of arrangements via spectral sequences. J. Comp. Syst. Sci. 67 (2003) 244-262.

[11] Danzer, L.: Three dimensional analogues of the planar Penrose tilings and quasicrystals. Discrete Math. 76 (1989) 1-7.

[12] Kramer, P.; Neri, R.: On periodic and non-periodic space fillings of $E^{m}$ obtained by projection. Acta Cryst A $\mathbf{4 0}$ (1984) 580-587.

[13] Kramer, P.; Papadopolos, Z.: Models of icosahedral quasicrystals from $6 \mathrm{~d}$ lattices. Proceedings of the International conference on Aperiodic Crystals, Aperiodic '94 (G. Chapuis et al., eds.), World Scientific, Singapore, 1995, pp. 70-76. 